\title{
Investigating Mobile Stereoscopic 3D Touchscreen Interaction
}

\author{
Ashley Colley ${ }^{1}$ \\ ${ }^{1}$ Center for Internet Excellence \\ University of Oulu \\ 90014 Oulu, Finland \\ firstname.lastname@cie.fi
}

\author{
Johannes Schöning ${ }^{2}$ \\ Maaret Posti ${ }^{1}$ \\ ${ }^{2}$ Hasselt University, tUL - iMinds \\ Expertise Centre for Digital Media \\ 3590 Diepenbeek, Belgium \\ firstname.lastname@uhasselt.be
}

\begin{abstract}
3D output is no longer limited to large screens in cinemas or living rooms. Nowadays more and more mobile devices are equipped with autostereoscopic 3D (S3D) touchscreens. As a consequence interaction with $3 \mathrm{D}$ content now also happens whilst users are on the move. In this paper we carried out a user study with 27 participants to assess how mobile interaction, i.e. whilst walking, with mobile S3D devices, differs from interaction with 2D mobile touchscreens. We investigate the difference in touch accuracy between 2D touchscreens and mobile S3D touchscreens and evaluate the minimum touch target size for mobile S3D touchscreens. The contributions of this paper are twofold: Firstly, we found the increase in minimum touch target size caused by walking was larger for a mobile S3D UI than for a 2D UI. Secondly, we present touch target sizes and aspect ratios required for reliable user interaction in each case. Additionally we examined differences in the angle at which users held the mobile S3D device compared to a 2D mobile device. We found that mobile S3D caused users to hold the device at a different angle when walking, compared to the $2 \mathrm{D}$ case. This first study of its kind provides valuable information to developers of the next generation of UIs and applications for mobile S3D displays and devices.
\end{abstract}

\section{Author Keywords}

Stereoscopy, 3D User Interfaces, Mobile User Interfaces, User Studies.

\section{ACM Classification Keywords}

H.5.m. Information interfaces and presentation (e.g., HCI): Miscellaneous.

\section{General Terms}

Human Factors, Design, Measurement.

Permission to make digital or hard copies of all or part of this work for personal or classroom use is granted without fee provided that copies are not made or distributed for profit or commercial advantage and that copies bear this notice and the full citation on the first page. To copy otherwise, or republish, to post on servers or to redistribute to lists, requires prior specific permission and/or a fee.

$\mathrm{OzCHI}$ '13, November 25 - 29 2013, Adelaide, Australia

Copyright 2013 ACM 978-1-4503-2525-7/13/11 ..\$15.00.

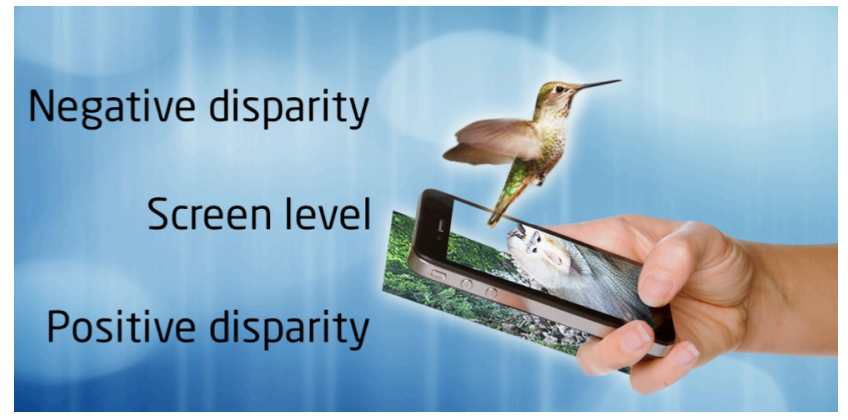

Figure 1. An autostereoscopic 3D mobile device. Content is displayed with negative (Bird), zero (Monkey) and positive (background image) disparity / parallax.

\section{INTRODUCTION}

Stereoscopic 3D (S3D) mobile devices are already massmarket products, and the penetration of such devices is growing in numbers. Autostereoscopic mobile 3D displays, where no special glasses (or similar devices) are needed to experience the 3D effect, can be found in 3D cameras (e.g. FinePix REAL 3D W3), mobile phones (e.g. LG Optimus 3D), and portable game consoles (e.g. Nintendo 3DS). In general 3D displays provide new degrees of freedom for UI design in the sense of the illusion of depth. With negative disparity (or parallax), the UI elements appears to float in front of the screen, and with positive disparity (or parallax), the UI elements appears behind the screen level (see Figure 1).

Valkov et al., (2010, 2011), identified the challenges of combining touch screen input with large 3D displays, and identified methods by which users' adapt to work around these. Despite these limitations, S3D displays are becoming more commonplace in a variety of mobile touch-screen devices. In order to introduce novel designs for mobile S3D UIs, we need to investigate the implications of mobile S3D devices on the user interaction (e.g. in terms of touch accuracy and resolution). We conducted studies not just in a static context, but also with users on the move, as it is an inseparable part of the usage of mobile technology. Other researchers have proven that mobile use of a mobile device affects the interaction with the device (BergstromLehtovirta et al., 2011; Oulasvirta et al., 2005). 
Additionally, there have been proposals that object depth within S3D UIs should be considered as an informative parameter (Häkkilä et al., 2012; Sunnari et al., 2012), and using S3D, e.g. for grouping items or highlighting contextual information, has been suggested (VentäOlkkonen et al., 2013). However, this work has generally not considered the usability of such designs beyond the static laboratory environment (Please note, later referrals to $\mathrm{S} 3 \mathrm{D}$ in this paper, if not specified in detail, refer to mobile S3D).

In this paper, we examine the utility of a mobile S3D touchscreen UI for practical usage when the user is on the move, and provide recommendations to developers and designers of such UIs. Specifically we investigated the effect of walking on two aspects that form the core a mobile S3D touch screen user experience: The perception of depth, and the accuracy of touching targets on the screen. We also compare the results achieved on a mobile S3D device with standard 2D mobile device, thus extending the existing body of research on mobile touch screen interaction on the move (Bergstrom-Lehtovirta et al., 2011; Kane et al., 2008).

As a contribution of this paper, we:

- Provide the first systematic analysis of the differences in interaction between 2D and 3SD mobile touch screens.

- Extend the boundaries of research on mobile touch screen interaction on the move from $2 \mathrm{D}$ to $\mathrm{S} 3 \mathrm{D}$.

- Report, to the advance of 2D, a significant difference in subjective perception of comfort when interacting with S3D and 2D mobile devices

- Introduce design recommendations for size and aspect ratio of touch target areas for mobile S3D devices.

\section{RELATED WORK}

HCI research on S3D has so far focused much on visual ergonomics, and visual comfort has been investigated in several studies (see e.g. Kooi and Toet, 2004; Lambooij et al., 2007; Pölönen et al., 2011). So far, interaction with S3D mobile devices has received less attention. When investigating mobile S3D UIs, the emphasis has been on the output rather than on interaction. Pölönen et al., (2011), report that the perceived depth is less sensitive to changes in the ambient illumination level than perceived naturalness and overall image quality. Mizobuchi et al., (2008), report that S3D text legibility was better when text was presented at zero disparity on a sinking background when compared to presenting it hovering on a background image that was presented in zero disparity. It has been also pointed out that scaling the S3D content to different size displays is not straightforward, and has an effect to the perceptual qualities (Benzeroual et al., 2012). The role of visual cues has been investigated. In regard to that topic, Mikkola et al., (2010), reveal that stereoscopic visual cues with a mobile device work better than 2D ones. Huhtala et al., (2011), report that for a find-and-select task in a S3D mobile photo gallery, both the performance and subjective satisfaction were better when the stereoscopic effect was combined with another visual effect, dimming. Taking a wider view to S3D UI design, a few papers have investigated the user experience with S3D mobile devices. Jumisko-Pyykkö et al., (2011), studied the quality of experience with mobile S3D videos, and discovered that the quality of experience was constructed from the following: visual quality, viewing experience, content, and quality of other modalities and their interactions. Sunnari et al., (2012), evaluated the S3D mobile UX design of a commercial product with a user study, where 12 users compared the S3D and 2D menus of a S3D mobile phone. The S3D menu design was seen as visually pleasant and entertaining, but lacking in usability, and the participants had difficulties seeing any practical benefit of using stereoscopy. In order to gain other than just hedonistic value, stereoscopy should be incorporated to the mobile UI in a way that it improves not only the visual design, but also the usability (Häkkilä et al., 2012).

\section{Investigating Input Accuracy}

Whereas the illusion of depth is the distinguishing factor between S3D and conventional 2D mobile devices, it is also important to look at the input accuracy on the $x, y$ plane. In addition to distinguishing between the depths of objects, touch target selection accuracy is a key issue when interacting with a mobile touch screen devices. A comprehensive body of work exists examining the input accuracy of 2D touch screens, e.g. Holtz and Baudisch, (2012) and Parhi et al., (2006). Holtz's summary, that inaccuracy is largely due to a "parallax" artifact between user control based on the top of the finger and sensing based on the bottom side of the finger is particularly relevant, as in the S3D case another on-screen parallax effect is introduced. The use of touch on large 3D touch screens, as mentioned earlier, was studied by Valkov et al., $(2010,2011)$. The effect of walking on touch accuracy for 2D touch screen UIs has also been previously researched (Bergstrom-Lehtovirta et al., 2011; Kane et al., 2008; Perry and Hourcade, 2008). For example, Kane finds variation in the optimal button size for different individuals, whilst Perry and Hourcade conclude that that walking slows users' interaction but has little direct effect on their touch accuracy. However, to best of our knowledge, earlier research has not examined the touch input accuracy with a S3D mobile device while on the move.

\section{Advancements over Related Work}

Taking the strong emphasis on visual design in S3D mobile products and the output orientated prior art, there is a clear need to further investigate the interaction design aspects of S3D mobile UIs. In this paper, we focus on assessing two aspects of interaction with a S3D mobile touch screen device when walking - distinguishing the depth of objects in the stereoscopic UI, and the target selection accuracy of objects at different depths and positions. Our aim is to 
provide practical information to assist the designers of $\mathrm{S} 3 \mathrm{D}$ UIs that will be used on the move.

The users' ability to assess the relative depth of objects in a S3D UI is critical in order to convey information in the depth dimension of the UI, rather than use the effect only for aesthetic purposes. Clearly, when encoding information to the perceived depth of objects, it should also be perceivable whilst the user is walking, and not only whilst static. Similarly, an understanding of the users' input accuracy when selecting targets in the mobile autostereoscopic UI is critical for the UI designer: At which depths should interactive components be placed? Which positions on the display are touched most accurately? What is the optimal size and aspect ratio of touch buttons?

This extends the current body of research which has focused either to evaluate S3D in static conditions, or interaction with a 2D UI whilst on the move; the combination of S3D and user motion being relatively unstudied.

\section{RESEARCH HYPOTHESES}

To systematically evaluate the problem area, we formulated the following hypotheses. Hypotheses $\mathrm{H} 1-\mathrm{H} 3$ focus on interaction while walking, which, due the cumulative effect of general challenges with stereoscopy (Lambooij et al., 2007) and movement, was expected to make the interaction with S3D relatively harder than with 2D UI.

H1: That the degradation in user's touch accuracy caused by walking will be greater for an autostereoscopic interface than a normal $2 D$ interface.

H2: Users will find the comfort level of using an S3D UI to be more adversely affected by walking than a $2 D U I$.

H3: Users' perception of the relative depths of objects in the $3 D U I$ will be reduced by walking to a greater extent than perception of relative object size.

H4: The accuracy of tapping objects on the S3D display will differ for objects at different locations on the display, and will be different in horizontal and vertical dimensions.

H5: In order to see the 3D effect when walking, users need to hold a device with an S3D display somewhat differently from a $2 D$ device.

\section{USER STUDY}

To validate the hypotheses, we conducted a user study with 27 participants.

\section{Technical Setup}

Our study consisted of two main parts, firstly focusing on the depth perception and secondly to the to the interaction accuracy (i.e. related to $x$ and $y$ perception and interaction with the touch screen). Together these two aspects define key aspects of the S3D touch screen interaction space. As we wanted to focus only on the depth effect due to the stereoscopy, no additional visual depth clues such as shadows, object size or color were used in our test application

\begin{tabular}{|l|l|l|}
\hline $\begin{array}{l}\text { Accuracy test } \\
\text { z-depths }\end{array}$ & $\begin{array}{l}\text { Depth test } \\
\text { z-depths }\end{array}$ & $\begin{array}{l}\text { Apparent distance behind } \\
\text { screen }(\mathrm{mm})\end{array}$ \\
\hline 10 & 10 & 12 \\
\hline 3 & Not used & 3.6 \\
\hline 2 & 2 & 2.5 \\
\hline Not used & 1 & 1.2 \\
\hline 0 & 0 & 0 (on screen) \\
\hline-1 & -1 & -1.2 (in front of screen) \\
\hline-2 & -2 & -2.5 (in front of screen) \\
\hline
\end{tabular}

Table 1. Reference stereoscopic depths and calculated apparent distance behind the display (assuming viewing distance of $330 \mathrm{~mm}$ and inter-pupil distance of $63 \mathrm{~mm}$ )

In all the tests a background wallpaper image was used, as in pilot tests the use of some type of textured background at the positive disparity/parallax (Figure 1) was found to improve 3D perception. The background image chosen was a fine mesh pattern at $45^{\circ}$, this was chosen as it gave good 3D perception, but was such that it would not adversely influence the position of the users' presses on targets. In the $2 \mathrm{D}$ tests the background was positioned at $\mathrm{z}$-depth $=$ zero and in the $3 \mathrm{D}$ tests at $\mathrm{z}$-depth $=10$. Table 1 describes the $\mathrm{z}-$ depth convention and values used in the study. All the tests were conducted on an autostereoscopic touchscreen mobile device, the LG P920 Optimus 3D mobile phone. This has a 4.3" display with a resolution of 480x800 pixels (with 217 ppi) and runs Android 2.3.5.

\section{Accuracy test}

Our target was to investigate the fundamental accuracy of users, hence our method avoided the use of any UI elements such as buttons, whose visuals could influence the position in which users tapped the screen. Additionally, as we were interested to investigate differences in accuracy between horizontal and vertical dimensions, a method based on measuring hit rates and task completion times for visual buttons would demand a large number of button shapes and sizes. Instead, we followed a method similar to the crosshairs approach employed by Holtz and Baudisch, (2012). Our accuracy test method presented rather small circular targets, one at a time. The user was instructed to tap on the center of each target with their index finger.

For each tap the coordinates of both the press and release touch events were logged, as well as the time between the target being displayed and the user pressing the target. Hence this method provides the touch event distributions for a point visual target. Additionally the time between the press and release events was logged. At the release event, when the user's finger was lifted from the screen, the values of the devices 3-axis accelerometer were logged, enabling 
information about the physical angle and movement of the device during the test to be deduced.

The accuracy test was conducted in the 2D and S3D condition. In the $2 \mathrm{D}$ condition a random sequence of 15 targets was presented. Each target was presented once and once only. The targets were positioned in a grid pattern of 5 horizontal by 3 vertical. This pattern was chosen to give more data points and resolution in the horizontal axis, which is the most interesting for S3D. The targets and background image were displayed as normal 2D objects.

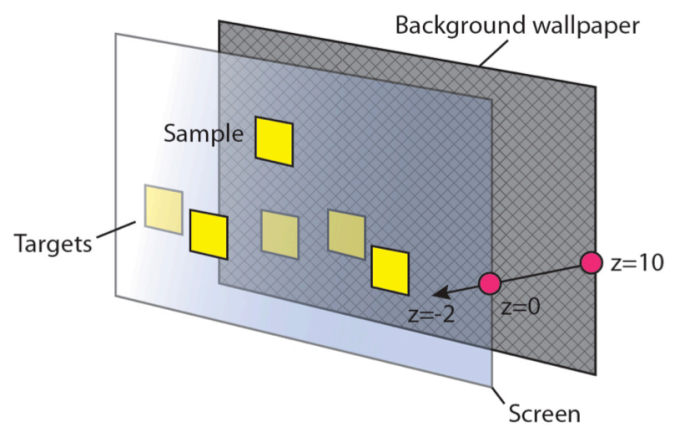

Figure 2. Diagram of the S3D depth perception test and depth convention used in the study.

In the $\mathrm{S} 3 \mathrm{D}$ version of the accuracy test the same grid of 15 targets as used in the 2D test was used, but it was positioned at 5 different z-depths (see Table 1), thus making a total of 75 targets. The 75 targets were presented in a random order of $\mathrm{x}, \mathrm{y}$ and $\mathrm{z}$ position, such that each target was presented once and once only. In the S3D test the background wallpaper image was positioned at depth $\mathrm{z}=10$, behind the screen. It should be noted that the due to the difference in background depth the S3D test with targets at $\mathrm{z}=0$ was not exactly equivalent to the $2 \mathrm{D}$ test.

Based on an eyes-to-screen distance of 330mm (the mean of 3 pilot users) and an inter-pupil distance of $63 \mathrm{~mm}$ (see Dodgson, 2004) the object distances in front of and behind the screen are given in Table 1. These values give an approximation of the apparent depth of objects presented at the different reference depths (the distance of the screen from the eyes was not fixed during the tests).

\section{Depth and Size Perception Test}

To test the users' perception of the z-depth of objects in the S3D display a depth-matching test was devised. A sample square object was drawn on the display with a random zdepth, selected from 5 possible depths (see Table 1). Five identical square target objects were also drawn to the screen each with a different depth (Figure 2). The order of the depths of the target objects was randomized. The user was instructed to select, by tapping, the target square object that they considered was at the same depth as the sample object. This is similar to the approach used by Mikkola et al, (2010).
As a comparison, a $2 \mathrm{D}$ size perception test was also devised. This used exactly the same process as the S3D depth perception test, but instead of varying the depth of the objects, the size of the square objects was varied slightly. The amount of size variation was determined during a pilot test to be approximately as challenging as the $3 \mathrm{D}$ depth perception test when static. Squares with sides of $4.2 \mathrm{~mm}$, $4.4 \mathrm{~mm}, 4.7 \mathrm{~mm}, 4.9 \mathrm{~mm}$ and $5.2 \mathrm{~mm}$ were used. Figure 3 shows the size comparison test.

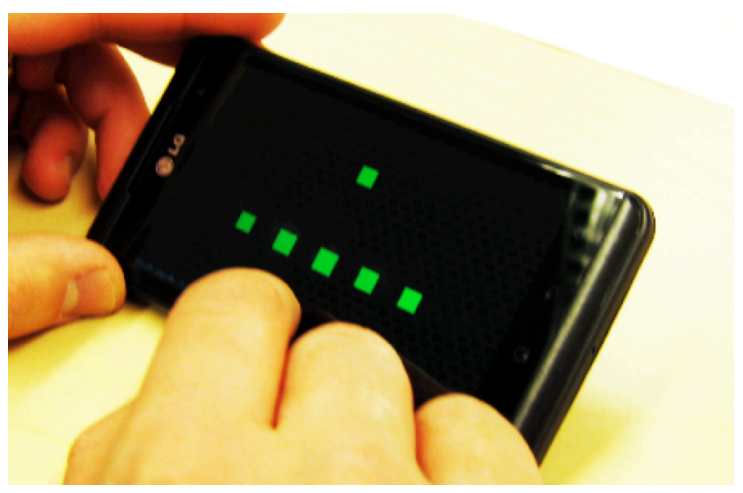

Figure 3. The static 2D size comparison test.

Both the depth and size perception tests were repeated 21 times, after which the user was presented with the score of how many depths or sizes they had correctly matched. All the tests were carried out whilst seated and while walking, with the order of tests completed by each user being varied as described in the user test procedure paragraph.

\section{Procedure}

The overall procedure was as follows

- Background questionnaire

- Tests for the dominant eye and stereovision

- Interaction tests: 2D accuracy, S3D accuracy, depth matching (S3D) and size matching (2D)

Each test was conducted in two different conditions; while seated (static) and whilst walking (on the move). After each test a Likert scale subjective rating was completed by the users. The presentation order of the conditions was counterbalanced between participants.

The test to identify the user's dominant eye was performed using the Portas method (see Kommerell et al., 2003). An acclimatization task of looking through the S3D image gallery, provided as default by the device manufacturer, enabled users to get accustomed to the S3D effect. Users were instructed to experiment to find the best position i.e. distance from device to eyes, viewing angle etc. where they could best see the 3D effect of the gallery images. For the walking tests, a route consisting of two markers $6 \mathrm{~m}$ apart was marked on the laboratory floor. Users were instructed to walk at normal speed around the marked route whilst completing the tests. 


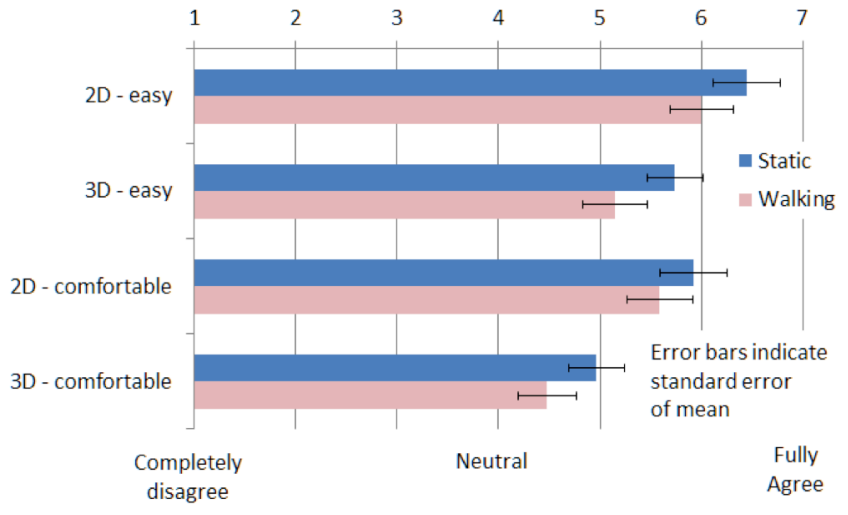

Figure 4. Subjective user feedback on accuracy tests.

The order of tests completed by each user was rotated in a sequence to reduce any effect of user learning or other side effects. After each test the users rated the test condition for easiness and comfort on a 7 point Likert scale.

\section{RESULTS}

\section{User Data}

The average age of the users $(n=27)$ was 30.4 years (varying from 10 to 52 ). 19 of the users were male and 8 were female. Of the 27 users 16 were found to have a dominant right eye whilst 9 had a dominant left eye. Two users were unable to determine which of their eyes was dominant. 24 of the users were right handed and the remaining 3 left handed. Most had watched a 3D movie, half had tried out, or own a 3D TV or a 3D camera.

\section{Accuracy Tests}

\section{Subjective Ratings}

The users' subjective opinions of the easiness and comfort of the 2D and S3D accuracy tests are shown in Figure 4. Two-way within subjects ANOVA tests were conducted. For "ease" the walking had a significant effect $(\mathrm{F}(1,26)=$ $10.95, \mathrm{p}=.003)$, making the task significantly harder when walking than when static. Also, completing the task using the S3D display was considered significantly harder than with the $2 \mathrm{D}$ display $(\mathrm{F}(1,26)=18.33$, $\mathrm{p}<$ $.001)$. There was no significant interaction between the walking state and display dimensionality $(\mathrm{F}(1,26)=.61, \mathrm{p}=$ .443). For "comfort" the results were similar, both walking and S3D made the task significantly less comfortable, $(\mathrm{F}(1,26)=4.75, \mathrm{p}=.039)$ and $(\mathrm{F}(1,26)=28.00, \mathrm{p}<0.01)$, respectively. There was no interaction between the independent variables $(\mathrm{F}(1,26)=1.94, \mathrm{p}=.663)$.

\section{Measurement of Input Accuracy}

Analysis of the accuracy tests is based on the recorded coordinates of the touch press event (i.e. "finger down"), as this defines the users' fundamental accuracy. Presses that were more than $12 \mathrm{~mm}$ from the center of the visual target were considered as accidental and excluded from further
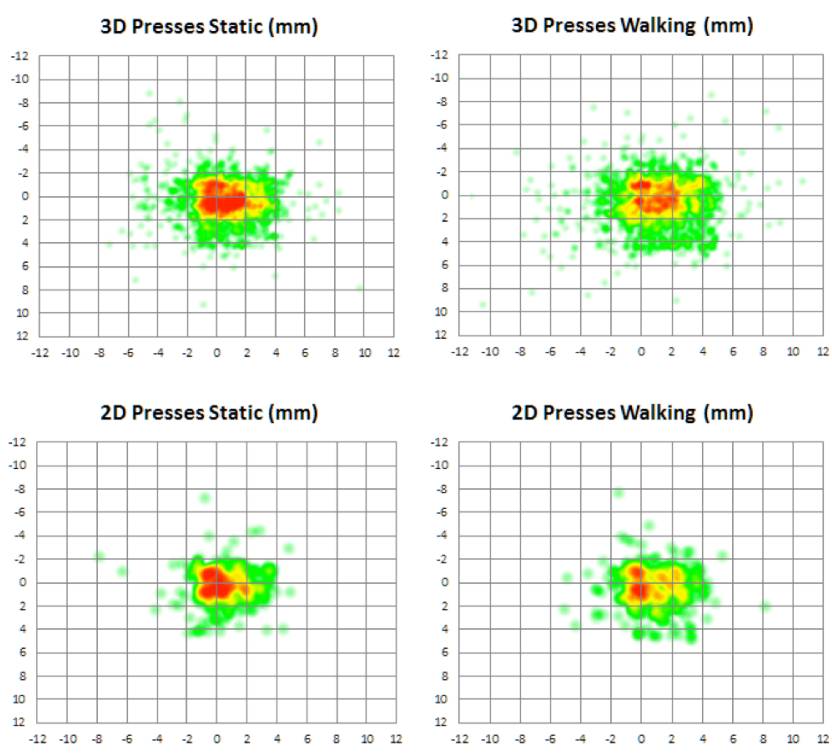

Figure 5. Heatmap visualizations of the combined press points relative to target center for $\mathrm{S3D}$ and $2 \mathrm{D}$.

analysis (Removed data: static $2 \mathrm{D}=2,3 \mathrm{D}=4$, walking $2 \mathrm{D}=3$, $3 \mathrm{D}=10)$.

Heatmaps of the press points for each test are shown in Figure 5, which combines the presses for all of the depth layers. These charts plot the offset of each press point from the center of the presented circular target, i.e. the center of the visual target is at the origin of the chart.

To investigate if there was any difference in the errors at different positions on the display (H4) we calculated the mean press position per presented target, illustrated in Figure 6. It can be seen that for all targets users are pressing slightly below and to the right. Contrary to the hypothesis, the target location on the display was not found to have an effect to the input accuracy.

The absolute distance of each press point from the center of the visual target (i.e. ignoring the direction of the error) is presented in Figure 7. For the S3D layers (i.e. within the groups of five bars in Figure 7), there was no significant difference between the mean error distance for each of the 3D depth layers (based on ANOVA analysis). Thus, targets at screen level $(\mathrm{z}=0)$ were pressed no more accurately than targets at other depths. For all the S3D depth layers combined together, a 2-way ANOVA test revealed both walking and S3D caused a significant increase in the mean error distance, $(\mathrm{F}(2,4841)=28.8, \quad \mathrm{p}<.001)$ and $(\mathrm{F}(2,4841)=54.9, \mathrm{p}<.001)$, respectively. There was no significant interaction between the fixed factors. 


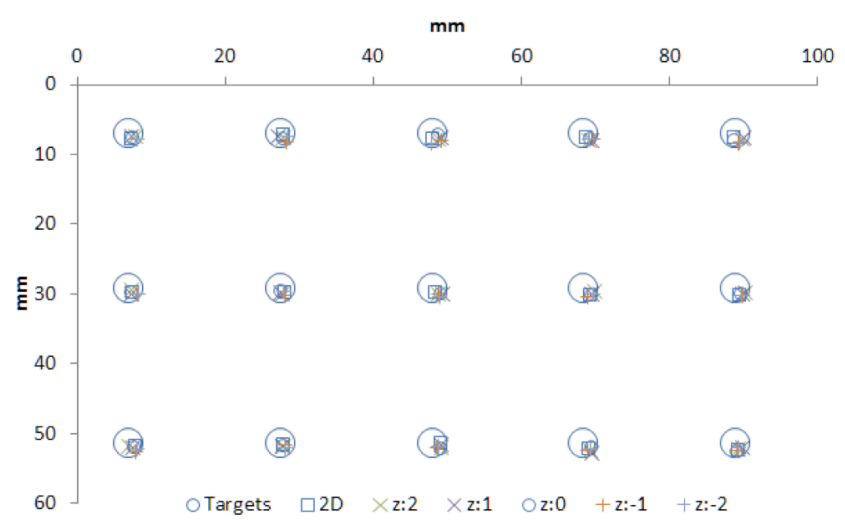

Figure 6. Mean press positions per target illustrated. The targets (marked here as circles) are shown larger than the original size used in the test for clarity.

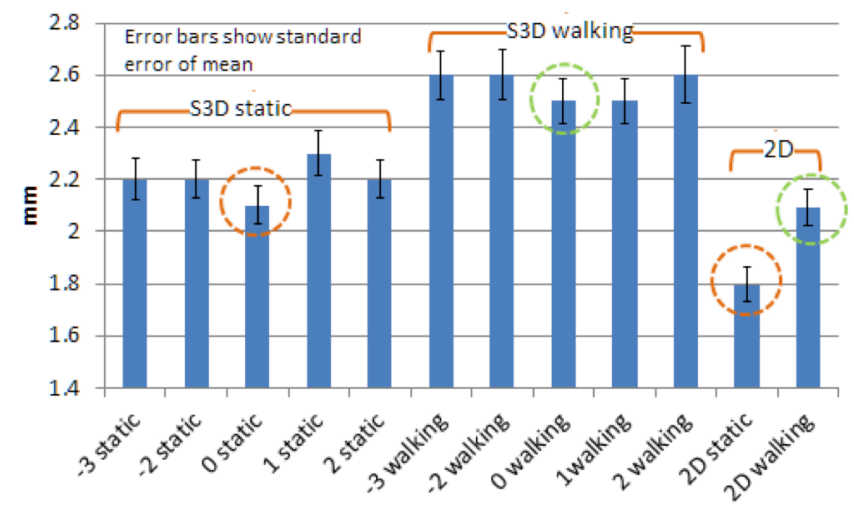

Figure 7. Mean distance from target center for 2D \& individual 3D layers whilst static and walking. Error bars show standard error of mean.

\begin{tabular}{|l|l|l|l|}
\hline Conditions & $\begin{array}{l}\text { Negative } \\
98 \\
\text { percentile } \\
(\mathrm{mm})\end{array}$ & $\begin{array}{l}\text { Positive } \\
98 \\
\text { percentile } \\
(\mathrm{mm})\end{array}$ & $\begin{array}{l}\text { Minimum } \\
\text { target size }(95 \% \\
\text { presses on target) }\end{array}$ \\
& $\begin{array}{l}\text { width } \mathrm{x} \text { height } \\
(\mathrm{mm})\end{array}$ \\
\hline 2D Static & $\begin{array}{l}\mathrm{x}: 3.5 \\
\mathrm{y}: 3.9\end{array}$ & $\begin{array}{l}\mathrm{x}: 3.8 \\
\mathrm{y}: 4.4\end{array}$ & $7.6 \times 8.8$ \\
\hline 2D Walking & $\mathrm{x}: 3.6$ & $\mathrm{y}: 4.2$ & $8.4 \times 9.2$ \\
& $\mathrm{y}: 3.7$ & $\mathrm{y}: 4.6$ & \\
\hline 3D Static & $\mathrm{x}: 4.9$ & $\mathrm{x}: 4.5$ & $9.8 \times 9.2$ \\
& $\mathrm{y}: 3.8$ & $\mathrm{y}: 4.6$ & $12.0 \times 10.4$ \\
\hline 3D Walking & $\mathrm{x}: 6.0$ & $\mathrm{x}: 5.0$ & $\mathrm{y}: 5.2$ \\
& $\mathrm{y}: 4.1$ & \multicolumn{2}{|l}{} \\
\hline
\end{tabular}

Table 2. Minimum target sizes for finger usage.

Comparing the $\mathrm{S} 3 \mathrm{D} \mathrm{z}=0$ results, where the target is placed at screen depth, with the corresponding 2D cases, where the target is also at screen depth (circled on Figure 7), showed significant degradation in accuracy in the S3D cases in comparison to 2D cases for $\mathbf{z}=\mathbf{0}(\mathrm{T}(403)=3.52, \mathrm{p}<.001$ and $\mathrm{T}(402)=4.35, \quad \mathrm{p}<.001$ for static and walking cases respectively). This difference is due to the $\mathrm{S} 3 \mathrm{D}$ cases having a background image at positive disparity, placing the targets within a stereoscopic scene. Additionally, in the $\mathrm{S} 3 \mathrm{D}$ cases, the $\mathrm{z}=0$ targets are presented in a sequence of targets at other depths. These findings are important as they indicate that from the touch accuracy point of view, there is no benefit to place targets at screen depth compared to any other depth level. Rather, to maximize touch accuracy, it is recommended that if $3 \mathrm{D}$ is not beneficial for other purposes within a UI, it is turned off.

\section{Minimum touch target sizes}

One method to evaluate the degradation in accuracy caused by walking and S3D is to calculate the minimum touch target sizes for each case. The minimum touch target size is the size required for users' to reliably hit touch targets in each tested mode. From the press point error distribution we calculated the 98 percentile points in positive and negative directions for both $\mathrm{x}$ and $\mathrm{y}$. Taking the approach described by Parhi et al., (2006), this defines the bounding rectangle that captures $95 \%$ of user presses. As the distributions are slightly skewed to either positive or negative sides, and in practice it is not possible to position a touch target at the exactly the correct offset from the center, thus we take double the larger absolute value, which may be considered as the minimum size for targets in the UI. It should be noted that these values are based on index finger usage, as in the test protocol. For thumb usage sizes will be somewhat larger (see Parhi et al., 2006). The minimum target sizes are shown in Table 2, and diagrammatically in Figure 8.

\section{Depth and Size Matching Tests}

Subjective Results

Figure 9 shows the results from the subjective questionnaire completed after each test. The size (2D) and depth (S3D) tests were found to be approximately equal in difficulty (subjectively "easy"). Two-way within subjects ANOVA tests revealed that walking significantly decreased the comfort level $(F(1,26)=5.10, p=.032)$. There was no significant effect of walking on "ease" or of S3D on "comfort" or "ease".

\section{Measurements}

The percentage of correct selections for both size and depth matching tests in both static and walking modes is shown in Figure 10.

When static, the users' performance in the depth and size matching tests was found to be approximately equal (this being the basis of the test design, based on the pilot test). However, we found that there was an extremely wide variation in performance between individual users, the standard deviations being 16 and 24 percentage points for size and depth comparison respectively (see Figure 11). Additionally, we were somewhat surprised that the mean error rate was quite high, close to $35 \%$. 


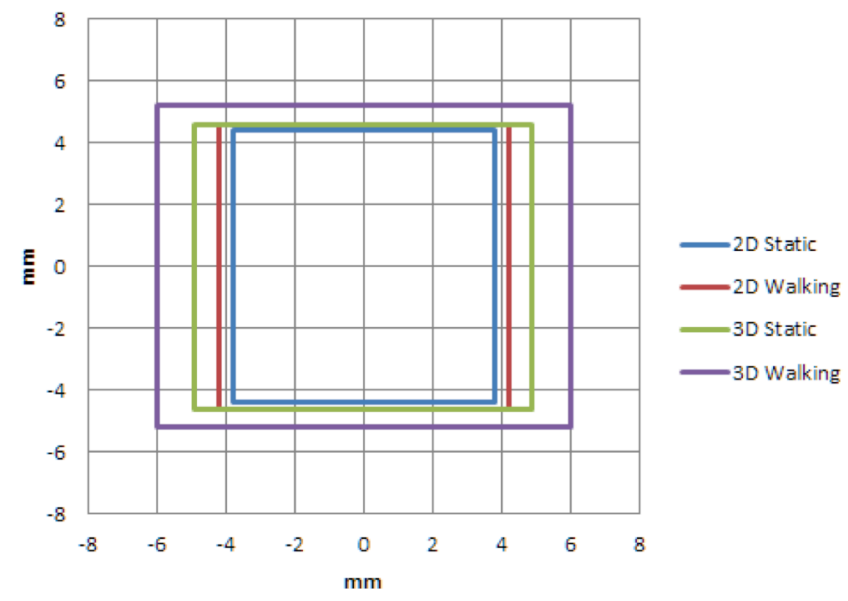

Figure 8. Minimum target sizes for finger usage $(95 \%$ press success)

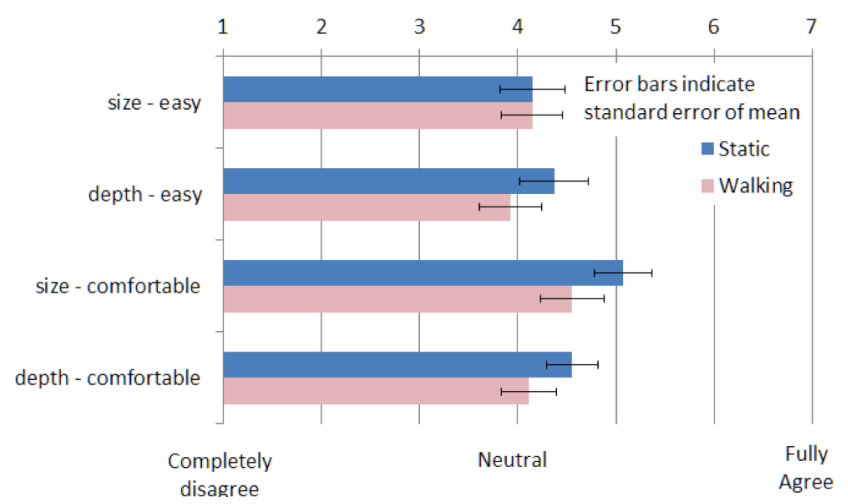

Figure 9. Subjective user feedback on size and depth tests.

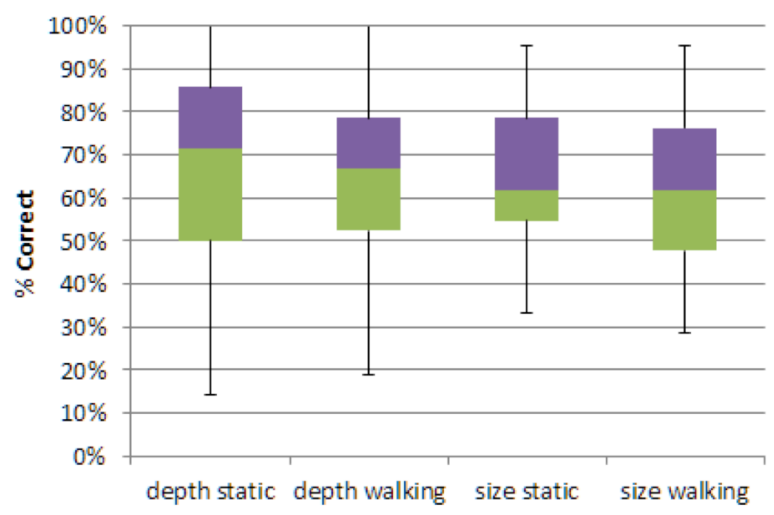

Figure 10. Percentage of correct selections for size and depth matching tests in static and walking test modes (showing quartile distribution).

Two-way ANOVA testing indicated no significant differences between the test cases. This is clearly due to the high standard deviation and limited sample size. There was general agreement between the measured performance and the users' subjective assessment of the tests difficulty ("easy"), i.e. all the tests were approximately equal in reported and measured difficulty.

\section{Device Orientation}

From the recorded accelerometer readings we extracted the mean orientation that the users were holding the device at during each test. The most interesting angle is the angle derived from the $\mathrm{z}$-axis of the accelerometer, the $\mathrm{z}$-angle (Figure 11).

For both depth (S3D) and size (2D) tests the angle at which the users held the device was larger when walking, i.e. the plane of the screen was closer to parallel with the ground (Figure 12). The mean angle of the device during the 2D tests was less than for the corresponding S3D tests. ANOVA testing indicated a significant effect of the test condition on the device angle (Wilks' Lambda $=0.40, \mathrm{~F}$ $(3,24)=11.92, p<.001)$. Post-hoc t-tests using Bonferroni adjusted alpha levels of .0125 per test $(.05 / 4)$ indicated that when walking, S3D caused a significant difference of approximately $7^{\circ}$ in the device angle, compared to $2 \mathrm{D}$ (Figure 13).

\section{Hypotheses Validation}

Hypothesis 1. Our hypothesis (H1) that the degradation in accuracy caused by walking is greater for an autostereoscopic interface than a 2D interface was found to be valid (Figure 7).

Hypothesis 2. We were able to partly validate our hypothesis related to the comfort level of S3D interaction whilst walking (H2). Whilst users found tasks requiring accuracy harder and more uncomfortable in the S3D case, tasks requiring visual comparison were not considered more challenging. Thus we find partial confirmation for our hypothesis that the absolute interaction comfort of an S3D UI when walking is lower than for a comparable 2D UI.

Hypothesis 3. We predicted that users' perception of the relative depths of objects in the S3D UI would be reduced by walking to a greater extent than perception of relative object size (H3). We found no evidence to support this based on our study.

Hypothesis 4. As part of our hypothesis (H4) we proposed that users' accuracy would differ in horizontal and vertical dimensions. Our study found a minimum touch target size aspect ratio of approximately 1.2 (Figure 8) confirming our prediction. This compares with the $2 \mathrm{D}$ case, where the minimum target aspect ratio was found to be roughly 1.0 (Figure 8). Based on our study, we were unable to validate the part our hypothesis (H4) that proposed accuracy differences at different locations on the S3D display (Figure $6)$.

Hypothesis 5. Our hypothesis that the users' would handle a device with an S3D UI differently from a device with a 2D UI when walking (H5) was partially confirmed. We 


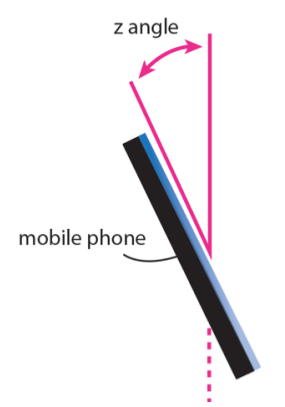

Figure 11. z-angle of the device.

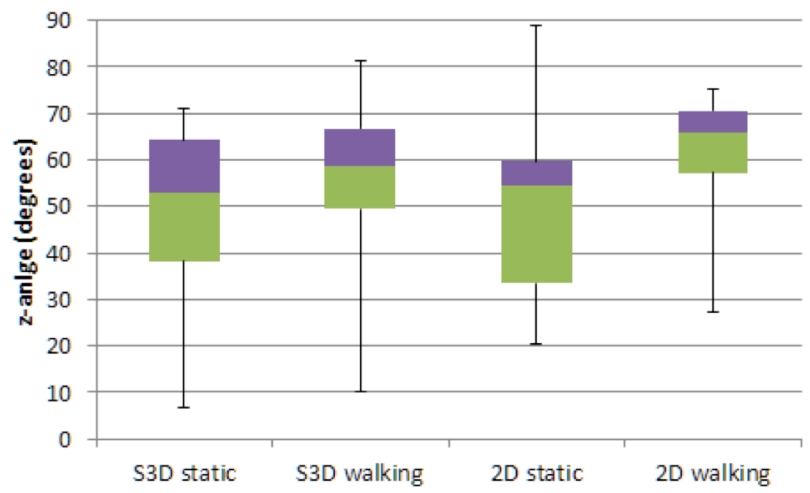

Figure 12. Quartile distribution of mean z-angle of device per test mode.

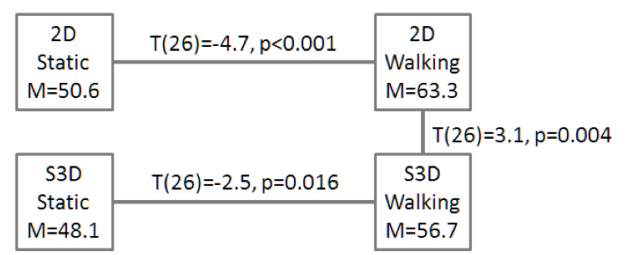

Figure 13. Significant differences in device z-angle between test cases (referring to Figure 12).

established that the mean angle that users' held the device when walking was different for 2D and S3D cases.

\section{DISCUSSION}

\section{Touch Accuracy}

Comparing our results for 2D static touch accuracy with previous studies, we find close agreement, for example, using their contact area model Holtz and Baudisch, report a $7.9 \mathrm{~mm}$ target size for $95 \%$ accuracy, compared to our $7.6 \mathrm{~mm} \times 8.8 \mathrm{~mm}$ for the same accuracy (Holtz and Baudisch, 2012). The aspect ratio information in our results provides interesting additional detail.

\section{The Effect of Walking on Accuracy}

Direct comparison between our results related to the differences in touch target sizes when walking with prior work on this topic is not straightforward, due to differences in the study approaches used. The majority of previous work being based on a UI with varied size button targets, and measuring task completion time and error rates (for example Kane et al., 2008). In contrast, our button free approach aimed to minimize the influence of the visual UI design, and focused to the accuracy of separate tap events. Thus, in our test, accuracy was the only dependent variable.

Our approach enabled us to investigate interaction over the full screen area, and we were able to gain insight into the optimal aspect ratio of touch targets. However, our method is more abstract than actual UI based approaches, requires some interpretation to transfer it to use in actual designs, and does not take account of other interaction issues e.g. related to Fitts' law (Fitts, 1954). Hence, it is perhaps the case that our method serves best as an initial method, the results of which could be validated by a button and task based method.

Based on our results, for a certain screen size, a S3D UI for use whilst walking should have only $60 \%$ of the touch targets of a correspondingly performing 2D UI (based on area calculations from Table 2 data). This is a major impact to the design of S3D mobile UIs.

The degradation in accuracy from the combined effects of S3D and walking appears to be larger than the sum of each individually (Figure 8). As may be expected the main part of this degradation is in the horizontal dimension, and hence related to stereoscopic effects. We speculate that, at least in part, this effect is due to users losing the stereoscopic effect, for example when glancing from the display when walking, which is an expected condition for real world interaction in mobile context (see Oulasvirta et al., 2005).

The Effect of Target Depth and Position on Accuracy

Our finding that there was little difference in the users' accuracy at different positions on the device screen differs to the systematic position related offsets reported by Henze et al., (2011). However, as proposed by Henze, this effect is perhaps related to the visual size of touch targets, and hence not applicable to our small targets.

Valkov et al., (2010, 2011), has reported touch offsets based on target depth. However, as one factor affecting this is the user's dominant eye it was not surprising that we were hot able to identify such differences without separating test subjects with left and right eye dominance in our analysis. We may also speculate that this phenomenon is not relevant to small size display and the limited depth capabilities of the device and technology used in our tests (Figure 7).

\section{Ergonomic Issues}

We were unable to find comparable research on details of how users' hold mobile devices whilst walking, although many of the issues are touched upon by Kane et al., (2008). 
In addition to these, issues related to the limited viewing angles of current S3D displays are well known. In our study users held the test device at a different angle for the 2D and $3 \mathrm{D}$ tests (Figure 12). This suggests that in order to observe the $3 \mathrm{D}$ effect the users needed to modify the device angle compared to the way they would naturally hold the device for interacting with a 2D UI. Particularly when walking, this need to adjust the device angle becomes an additional cognitive, and perhaps physical, overhead whilst the user is already under a heavy cognitive load. This argues against the use of S3D UIs in mobile devices, or is something that should be addressed by device manufacturers.

\section{Usability Concerns with S3D}

Both accuracy and depth perception tests were such that they highlighted a large variation in the performance of individuals, which was further exaggerated when the users were walking. This large variation makes it difficult to identify statistically significant generic differences between the cases examined without a very large test sample. When designing any user interface it is not wise to design for the average user, without considering the variation of user performance. In the case of S3D UIs this suggests that the use of depth as a mandatory informative element in the UI should be approached cautiously. Clearly, when accessibility for users with restricted capabilities is a consideration, the use of $\mathrm{S} 3 \mathrm{D}$, at least as an informative channel should be avoided.

\section{Future Work}

Interestingly, visual examination of the distribution of presses (see Figure 5) suggests a slight shift to the right whilst walking. This appears similar for both $2 \mathrm{D}$ and S3D cases. The reason for this is unknown and would require further study. Possible causes could be differences in the distance between the users' eyes and the screen, or differences in the viewing angle. Such small differences may prove relevant for tasks requiring the pressing of very small targets, such as on screen QWERTY keyboards used for text input, whilst walking.

The current study was rather limited in its investigation of differences in the way users hold the device whilst static and walking, for 2D and S3D. Device angle was recorded only at the time the screen was pressed and only the average angle was analyzed. It would be interesting to investigate the amount of movement during the task, and to relate this to the different physical approaches (grip, device position, etc. employed by users to maximize their performance in using the device whilst walking.

\section{CONCLUSIONS}

We found a very large variation in the perception of S3D object depths between users. The variation in perception of 2D object size was also large, but somewhat less than for depth. Both size and depth perception were similarly degraded by walking. Additionally, when walking, users held the device differently when using a S3D UI vs. a 2D $\mathrm{UI}$, the mean device angle being $57^{\circ}$ for $2 \mathrm{D}$ vs. $63^{\circ}$ for
S3D. This wide variation in user performance, and additional interaction overhead, should be considered when designing S3D UIs.

The mean press positions on visual targets at all z-depths on a S3D touch screen were almost identical, i.e. there was no discernible offset caused by placing targets at different zdepths (although our analysis was limited in that we did not separately analyze users based on their dominant eye). Additionally we found no effect of target location on the display on the users' accuracy in 2D or S3D cases. However, the variance in press accuracy in the S3D case was much larger than for 2D, and this difference was more pronounced when walking.

When in static usage, to achieve the same performance as 2D, S3D touch targets need to be horizontally wider (7.6mm for $2 \mathrm{D}$ vs. $9.8 \mathrm{~mm}$, for $3 \mathrm{D}$ for $95 \%$ presses on target). To support usage whilst walking, the size of the touch targets on a touch screen S3D user interface needs to be significantly larger than the corresponding minimum for a 2D interface. In our study the minimum sizes for $95 \%$ presses on target was $8.4 \mathrm{~mm} \times 9.2 \mathrm{~mm}$ (width $\mathrm{x}$ height) for $2 \mathrm{D}$ vs. $12.0 \mathrm{~mm} \times 10.4 \mathrm{~mm}$ for $3 \mathrm{D}$. Although these values are based on the test device used, it is expected that they should be generally applicable to other similar S3D devices. For example, the presented minimum touch target sizes in millimeters should serve as initial guidelines for UI designers of other S3D touch screen products intended for mobile use.

Our study provides practical guidance for the developers and designers of mobile stereoscopic 3D device UIs, both on the usage of depth to convey information, and on the touch target size required to interact with the UI. To summarize, the main findings of the paper are as follows:

- For input accuracy, both walking and S3D caused a significant increase in the mean error distance.

- Users' subjective perception of the input accuracy task was that using the S3D display was significantly harder than the 2D display, and was significantly harder when walking than when static.

- When walking, users hold the mobile device with S3D $\mathrm{UI}$ at a different angle in comparison to 2D UI.

- We have presented the minimum target size for S3D mobile touch screen interaction

\section{REFERENCES}

Benzeroual, K., Allison, R. S., Wilcox, L. M. 3D Display size matters: Compensating for the perceptual effects of S3D display scaling. In Proc. IEEE CVPRW, (2012), 45 -52 , IEEE. 
Bergstrom-Lehtovirta, J., Oulasvirta, A., Brewster, S. The effects of walking speed on target acquisition on a touchscreen interface. In Proc. MobileHCI, (2011), ACM.

Dodgson, Neil A. Variation and extrema of human interpupillary distance. Stereoscopic Displays and Applications. In Proc. SPIE, (2004), 5291: 36-46.

Fitts, P. M., The information capacity of the human motor system in controlling the amplitude of movement, Journal of Experimental Psychology, 47, (1954), 381-391.

Häkkilä, J., Kytökorpi, K., Karukka M.. Mobile Stereoscopic 3D User Experience - Calling for User Centric Design. In Proc. 3DCHI workshop at $\mathrm{CHI}$, (2012).

Hassenzahl, M. User Experience (UX): Towards an experiential perspective on product quality. In Proc. IMH, (2008), ACM, 11-15.

Henze, N., Rukzio, E. and Boll, S. 100,000,000 Taps: Analysis and Improvement of Touch Performance in the Large, In Proc. MobileHCI, (2011).

Holtz, C. and Baudisch, P. Understanding touch. In Proc. CHI, (2011), pp. 2501-2510.

Huhtala, J., Karukka, M., Salmimaa M. and Häkkilä, J. Evaluating Depth Illusion as Method of Adding Emphasis in Autostereoscopic Mobile Displays. In Proc. MobileHCI, (2011).

Jumisko-Pyykkö, S., Dominik Strohmeier, D., Utriainen, T., Kunze, K. Descriptive Quality of Experience for Mobile 3D Video. In Proc. NordiCHI, (2010), 266-275. ACM.

Kane, S., Wobbrock, J., Smith, I. Getting off the treadmill: evaluating walking user interfaces for mobile devices in public spaces. In Proc. MHCI08, (2008), 109-118.

Kommerell G, Schmitt C, Kromeier M, Bach M. Ocular prevalence versus ocular dominance. Vision Res. (2003); 43:1397-1403.

Kooi, F., Toet, A. Visual Comfort of Binocular and 3-D Displays. Displays 25, (2004), 99-108, 2004.
Lambooij, M. T. M., Wijnand A. IJsselsteijn, W. A., Heynderickx, I. Visual Discomfort in Stereoscopic Displays: A Review. Stereoscopic Displays and Virtual Reality Systems XIV, SPIE-IS\&T, (2007).

Mikkola, M., Boev, A., Gotchev, A. Relative Importance of Depth Cues on Portable Autostereoscopic Display. In Proc. MoViD at ACM Multimedia, (2010), 63-68.

Mizobuchi, S. et al. The Effect of Stereoscopic Viewing in a Word Search Task with a Layered Background. Journal of the Society for Information Display 16/11, 1105-1113, 2008, (2008).

Oulasvirta, A., Tamminen, S., Roto, V., Kuorelahti, J. Interaction in 4-second bursts: the fragmented nature of attentional resources in mobile HCI. In Proc. CHI, (2005), ACM.

Parhi, P., Karlson, A. K., and Bederson, B. B. Target size study for one-handed thumb use on small touchscreen devices. In Pro. MobileHCI, (2006), ACM.

Perry, K. and Hourcade, J. P. Evaluating One Handed Thumb Tapping on Mobile Touchscreen Devices. In Proc. Graphics Interface, (2008). p. 57-64.

Pölönen, M., Salmimaa, M., Häkkinen, J. Effect of ambient illumination level on perceived autostereoscopic display quality and depth perception. Displays 32, (2011) 135141. Elsevier.

Sunnari, M., Arhippainen, L., Pakanen, M., Hickey, S. Studying User Experiences of Autostereoscopic 3D Menu on Touch Screen Mobile Device. In Proc. OZCHI, (2012), ACM.

Valkov, D., Steinicke, F., Bruder, G., Hinrichs, K., Schöning, J., Daiber, F., and Krüger, A. Touching Floating Objects in Projection-based Virtual Reality Environments. In Proc. EGVE/EuroVR/VEC, (2010), 17-24.

Valkov, D., Steinicke, F., Bruder, G. and Hinrichs, K. 2D Touching of 3D stereoscopic objects. In Proc. of $\mathrm{CHI}$, (2011), ACM, 1353-1362.

Ventä-Olkkonen, L., Posti, M., Häkkilä, J. How to Use 3D in Stereoscopic Mobile User Interfaces - Study of Initial User Perceptions. In Proc. Academic MindTrek. ACM (2013). 